\title{
Galleria mellonella (greater wax moth) larvae as a model for antibiotic susceptibility testing and acute toxicity trials
}

\author{
Katarzyna Ignasiak and Anthony Maxwell ${ }^{*}$ (D)
}

\begin{abstract}
Background: Infectivity trials and toxicity testing in rodents are important prerequisites to the use of compounds in man. However, trials in rats and mice are expensive and there are ethical considerations. Galleria mellonella (greater wax moth) larvae are a potential alternative. We have assessed the use of these insects in infectivity trials and toxicity testing.

Findings: Using four bacterial species (two Gram-negative and two Gram-positive) we have assessed the efficacy of four antibiotics against infections in Galleria and compared the antibiotic susceptibility with that in humans. In general, we find a good correlation. Similarly, we have assessed 11 compounds (initially tested blind) for their toxicity in Galleria and compared this with toxicity trials in mice and rats. Again we found a good correlation between toxicity in Galleria and that in rodents.
\end{abstract}

Conclusion: We have found, in our hands, that G. mellonella larvae can be used in infectivity trials and toxicity testing, and that these assays represent an inexpensive and readily executable alternative to testing in rodents.

Keywords: Galleria mellonella, Infectivity testing, Toxicity trials, Antibiotics

\section{Background}

It is of vital importance that compounds intended for use in humans are adequately tested in suitable animal systems. Rodents (rats and mice) are commonly used in this regard, but cost and ethics have to be carefully considered. For example, the 3Rs (Replacement, Reduction and Refinement) are increasingly seen as a framework for conducting high quality science in the academic and industrial sectors with more focus on developing alternative approaches that avoid the use of animals [1]. It is therefore useful to contemplate alternative invertebrate models, if these can be shown to yield useful data. The greater wax moth (Galleria mellonella) is an insect in the order Lepidoptera and its larvae have previously been used for virulence and antimicrobial efficacy studies [2]. There are many benefits to using wax-moth larvae. Many

*Correspondence: tony.maxwell@jic.ac.uk Department Biological Chemistry, John Innes Centre, Norwich Research Park, Norwich NR4 7UH, UK larvae can be used in each experiment making pharmacokinetic and pharmacodynamic data easy to obtain. Pharmacokinetic data obtained in G. mellonella, such as antibiotic clearance time, elimination half-time of the drug and maximum drug concentration, can directly correlate to human data [3]. Numerous studies confirm that microbial pathogenicity and virulence determinants are the same in humans, mice and wax moths [4-6]. The insects can be bred quickly (at $37{ }^{\circ} \mathrm{C}$ the full life cycle lasts $\sim 6$ weeks) at low cost and without the need for specialized equipment; in addition they are not generally subject to ethical considerations. Galleria larvae are large, reaching 250-300 $\mathrm{mm}$ in length at fifth instar, enough for intraperitoneal injection of test compounds. Additionally, the insect immune system is functionally and structurally similar to the mammalian innate immune system $[7,8]$.

Galleria mellonella larvae were first used to assess antibiotic efficacy against Acinetobacter baumannii [9]. Cefotaxime, tetracycline, gentamicin and meropenem were assayed against a systemic $A$. baumannii 
infection. Gentamicin and meropenem, which A. baumannii is susceptible to, significantly prolonged the survival of infected larvae, while the survival of infected but untreated larvae and larvae treated with cefotaxime and tetracycline, which the bacteria are resistant to, was less than $25 \%$ in 5 days. The model quickly gained interest with a number of academic groups using it as a standard testing model. G. mellonella larvae have been used to investigate emerging pathogens [10] and novel treatments for persisting pathogens [3].

Toxicity testing in G. mellonella is an extrapolation from antibiotic efficacy studies. Antibiotic efficacy studies establish a dose of an antibiotic necessary to clear a bacterial infection. One of the necessary controls in antibiotic efficacy testing is to ensure that the antibiotic itself is not toxic to the insects. Apart from establishing a safe dose for efficacy testing a $\mathrm{LD}_{50}$ dose (median lethal dose; a dose of compound that is sufficient to kill $50 \%$ of a population of test animals) can be measured. However only recently have G. mellonella larvae been used in de novo toxicity testing. The toxicity of ionic liquids, which are low temperature molten salts used as an alternative to volatile solvents, has been assayed [11]. Ionic liquids are commonly labelled "ecologically friendly", even though the class is diverse and exhibits a wide range of toxicities. In this study the systemic toxicity was correlated to the length of alkyl chains of the 1-alkyl-3-methylimidazolium ionic liquids tested. The salts were toxic under $100 \mu \mathrm{g} / \mathrm{g}$, apart from the shortest two-carbon alkyl chain salt (1-ethyl-3-methylimidazolium chloride) which had a $\mathrm{LD}_{50}$ of nearly $8000 \mu \mathrm{g} / \mathrm{g}$, presenting a negligible toxicity. Interestingly, even though their rising popularity as an alternative to volatile organic compounds, 1-alkyl3-methylimidazolium ionic liquids lack toxicity data.

The aim of the present work was to evaluate the effectiveness of G. mellonella in antibiotic susceptibility trials and to explore whether they could also be routinely used in acute toxicity assays. Specifically, for the antibiotic efficacy trials we wanted to establish if the therapeutic doses established in the wax moth larvae correlate with doses recommended for human use, and for the toxicity testing we wanted to determine if the $\mathrm{LD}_{50}$ values established for wax moth larvae correlate to values established in rodents.

\section{Methods}

\section{Bacterial strains and media}

We chose two Gram-positive and two Gram-negative bacterial strains that are of clinical relevance for these studies; these were: Escherichia coli (ATCC 25922), Mycobacterium smegmatis (ATCC 700084), Pseudomonas aeruginosa (ATCC 15692) and Staphylococcus aureus (ATCC 29213), and were obtained from the
Health Protection Agency culture collection (Public Health England, Porton Down, UK). They were cultured from glycerol stocks and maintained on appropriate media on agar plates [Middlebrook medium (BD Difco 7H9) for M. smegmatis, LB (LMM0202, Formedium) for all others] before growth in LB broth aerobically at $37^{\circ} \mathrm{C}$. Cultures were sub-cultured at least twice before being used in the assays.

\section{Compounds}

Ampicillin, ciprofloxacin, rifampicin and tetracycline for antibiotic efficacy testing were obtained from Sigma Chemicals. For toxicity testing of compounds supplied by Inspiralis Ltd., sources were: ciprofloxacin (Fluka), etoposide, novobiocin, amsacrine, $\mathrm{NaCl}$, tetracycline, DMSO, chloroquine, streptomycin and ATP (Sigma), chloramphenicol (Duchea Biochemie), doxorubicin (Calbiochem) and glucose (Fisher Chemicals) (Table 1). Due to insolubility issues, amsacrine was supplied at $8 \mathrm{mg} / \mathrm{ml}$ in $50 \%$ DMSO in water; doxurubicin was supplied at $5.5 \mathrm{mg} /$ $\mathrm{ml}$ in $50 \%$ DMSO in water. For toxicity trials all compounds were initially provided in numbered tubes without compound names to avoid bias. The identity of the compounds was revealed only when the testing procedure was completed, and the data from G. mellonella was compared to material safety data sheet (MSDS) pages available with the compounds.

\section{Insect rearing}

A colony of G. mellonella was obtained from the John Innes Centre Entomology Facility (originally sourced

\section{Table 1 Compounds used in this study}

\begin{tabular}{lll}
\hline Compound & $\begin{array}{l}\text { Stock } \\
\text { concentration } \\
(\mathbf{m g} / \mathbf{m l})\end{array}$ & Notes \\
\hline Ampicillin & 25 & Supplied in 50\% DMSO in water \\
Amsacrine & $8^{\mathrm{a}}$ & Supplied in 50\% DMSO in water \\
Chloroquine & 25 & Supplied in 10\% DMSO in water \\
Ciprofloxacin & 25 & Supplied in 10\% DMSO in water \\
DMSO & N/A & Negative control \\
Doxorubicin & $5.5^{\mathrm{a}}$ & Supplied in 50\% DMSO in water \\
Etoposide & 25 & Supplied in 50\% DMSO in water \\
Glucose & 25 & Supplied in 10\% DMSO in water \\
Novobiocin & 25 & Supplied in 50\% DMSO in water \\
Rifampicin & 25 & Supplied in 50\% DMSO in water \\
Sodium chloride & 25 & Supplied in 10\% DMSO in water \\
Streptomycin & 25 & Supplied in 10\% DMSO in water \\
Tetracycline & 25 & Supplied in 10\% DMSO in water \\
\hline
\end{tabular}

N/A not applicable

a Lower concentration due to insolubility 
from Livefood UK Ltd.). The colony was kept in the dark at $37{ }^{\circ} \mathrm{C}$ in large Petri dishes $(140 \mathrm{~mm}$, Sterilin) filled with artificial food. The artificial food was composed of $300 \mathrm{ml}$ honey (Sainsbury's Honey, Clear), $400 \mathrm{ml}$ glycerol (G5516, Sigma Chemicals), 200 g milk powder (Dried Skimmed Milk Powder, Marvel), 200 g wholemeal flour (Strong Stoneground 100\% Wholemeal Flour, Sainsbury's), 100 g yeast powder (103753, Merck), $100 \mathrm{~g}$ wheat germ (Neal's Yard Wholefoods Natural Wheatgerm) and $400 \mathrm{~g}$ bran (Neal's Yard Wholefoods Natural Wheat Bran). First, the dry and wet ingredients were mixed separately, and then the mixtures were combined. The diet was mixed with beeswax pellets at a 2:1 ratio. Unused food was stored at $4{ }^{\circ} \mathrm{C}$. The food was replaced at least once per week, unless not enough was left for the larvae to feed on, in which case more food was added to the containers.

\section{G. mellonella injection procedure}

Five to ten larvae (250-320 mg each) were selected at random for each step in the procedure. Any larvae with darkening of the cuticle were discarded. The test compounds were injected into the hemocoel in DMSO or PBS buffer through the last left proleg (Hamilton syringe $701 \mathrm{~N}$, volume $10 \mu \mathrm{l}$, needle size $26 \mathrm{~s}$, cone tip) [10] unless stated otherwise. The larvae were placed on medical tissues (Kimtech) to stop the hemolymph from leaking. The larvae were incubated in the dark for 5 days and mortality was recorded daily.

\section{Determination of the infective dose of bacteria}

An infective dose of bacteria was determined by injecting groups of five larvae with bacterial suspension at: $5 \times 10^{4}$ colony forming units (cfu) per injection, $5 \times 10^{5} \mathrm{cfu}$, $5 \times 10^{6} \mathrm{cfu}$ and $5 \times 10^{7} \mathrm{cfu}$. The larvae were incubated for 5 days. An infective dose was defined as one that caused an immune response, recognizable by the darkening of the cuticle [10]. In G. mellonella larvae immune response leads to the formation of melanin plaques around bacteria immobilized by the cells of the immune system. These plaques appear dark through the cuticle. An infective dose of bacteria was one that caused $60-80 \%$ lethality within $48 \mathrm{~h}$, but not $100 \%$ lethality within $24 \mathrm{~h}$. The larvae were incubated at $37{ }^{\circ} \mathrm{C}$ as bacterial virulence changes with temperature and the experiment was designed to mimic infection in humans.

\section{Antibiotic efficacy testing}

A flowchart was used to assign an antibiotic therapeutic dose against a panel of bacteria (Fig. 1). For the antibiotic efficacy experiment five larvae (as recommended by OECD guidelines) were injected into the last left proleg with a pre-determined infective dose of bacteria and incubated for $2 \mathrm{~h}$ at $37{ }^{\circ} \mathrm{C}[3,12,13]$. After the incubation the larvae were injected in the last right proleg with the lowest dose of antibiotic $(5 \mathrm{mg} /$ $\mathrm{kg}$ body weight) and returned to incubation at $37{ }^{\circ} \mathrm{C}$. The mortality was recorded daily for 5 days. If three or more larvae survived, the lowest dose was re-tested and the lowest antibiotic dose was assigned as therapeutic. If three or more larvae died, the antibiotic was tested against the same infective dose of bacteria at a higher dose $(25 \mathrm{mg} / \mathrm{kg}$ body weight). The experiment was continued until a therapeutic dose was assigned or an antibiotic was ineffective against the infection. The values obtained were compared to values recommended for human use [14]: ampicillin-50-200 $\mathrm{mg} / \mathrm{kg}$ body weight/day, ciprofloxacin-10-15 $\mathrm{mg} / \mathrm{kg}$ body weight/ day, tetracycline $-25-50 \mathrm{mg} / \mathrm{kg}$ body weight/day and rifampicin-10-20 mg/kg body weight/day. Each step in the procedure included three control groups: untreated control, traumatized control (cuticle was pierced with a needle) and buffer-injected control.

\section{Toxicity testing procedure}

A flowchart, adapted from the OECD guidelines for acute toxicity [15], was used to select the toxic dose of test compounds (Fig. 2). The acute toxicity testing was started by injecting five larvae with the initial dose of a compound ( $5 \mathrm{mg} / \mathrm{kg}$ body weight). Larval mortality was recorded daily. If three or more larvae died, the compound was assigned the highest toxicity class (GHS 1). If three or more larvae survived for 5 days, the toxicity testing was continued by re-testing the initial dose $(5 \mathrm{mg} / \mathrm{kg}$ body weight) on a new cohort of larvae. If three or more larvae of the second cohort survived, a higher dose $(25 \mathrm{mg} / \mathrm{kg}$ body weight) was tested in five fresh larvae. The experiment was continued until a toxic dose was established. If a compound was not toxic at the highest dose tested (2000 $\mathrm{mg} / \mathrm{kg}$ body weight), the compound was classified as non-toxic. The obtained toxic dose was compared to a dose reported in Material Safety Data Sheet (MSDS) for the compound. Where possible the reported value used for the comparison was from mouse or rat via an intraperitoneal injection, when such data was absent the data from oral toxicity tests in a mammalian system was used. Each step in the procedure included three control groups: untreated control, traumatized control (cuticle was pierced with a needle) and buffer-injected control.

\section{Results and discussion Study design}

Both the antibiotic efficacy trials and the toxicity testing were based on OECD guidelines for toxicity testing in mice and rats [15]. The guidelines have been adapted for the use with $G$. mellonella because they are a statistically 


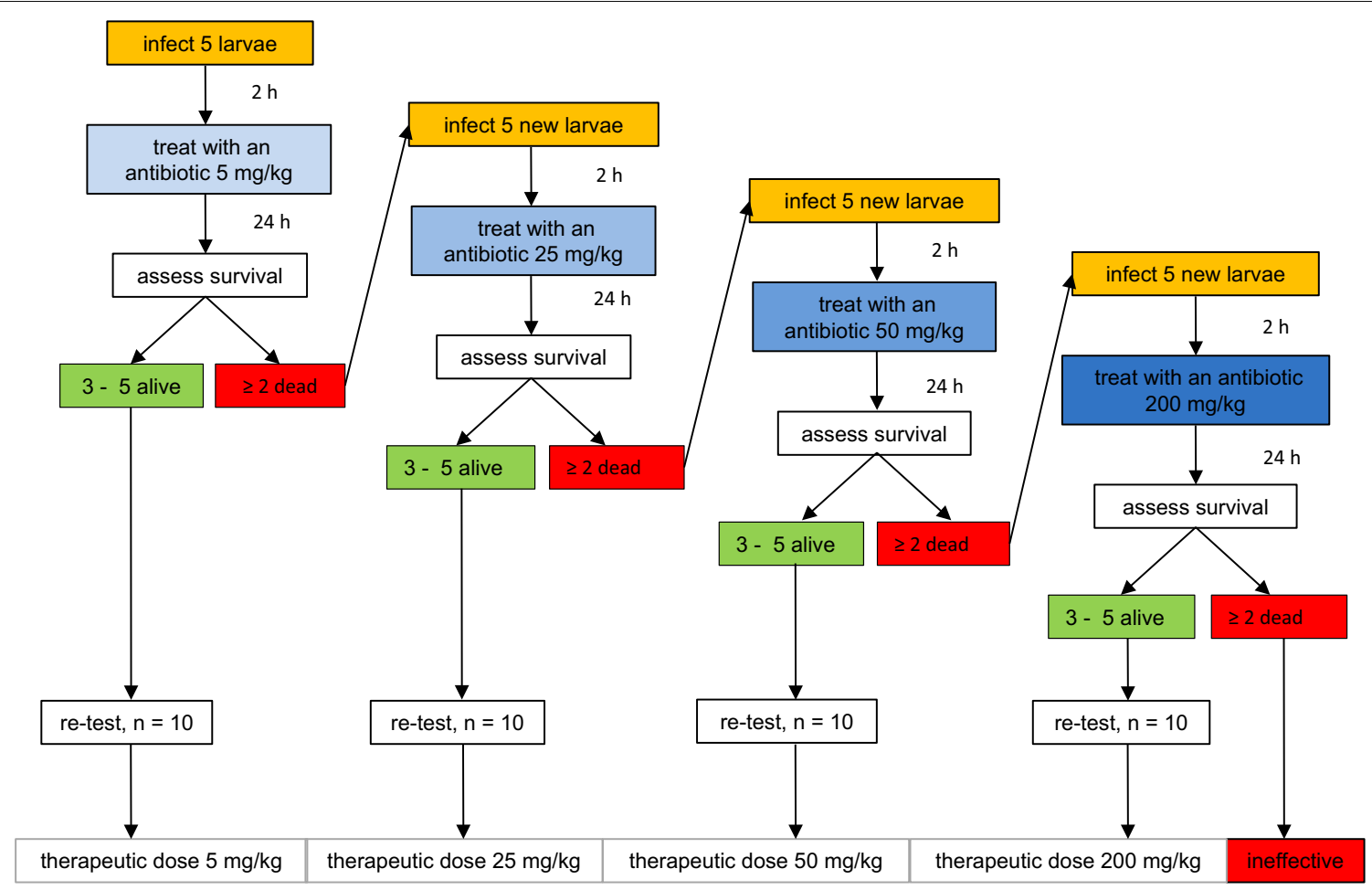

Fig. 1 A flowchart representing consecutive steps in the antibiotic efficacy test. A starting dose of $5 \mathrm{mg} / \mathrm{kg}$ body weight was administered and the insects were scored for survival. If the mortality was under $40 \%$, the compound was assigned the lowest therapeutic dose. If the mortality was over $40 \%$, a higher dose was tested until a therapeutic dose was established

robust method and allow for a streamlined workflow with a clear start and end point.

\section{Antibiotic efficacy}

Initially the appropriate infective dose of bacteria was determined for each bacterial species. An infective dose was one that caused immune response observed as darkening of the cuticle and $60-80 \%$ lethality within $48 \mathrm{~h}$, but not $100 \%$ lethality within $24 \mathrm{~h}$. This was determined to be $5 \times 10^{6} \mathrm{cfu}$ in $10 \mu \mathrm{l}$ for E. coli, M. smegmatis and $S$. aureus, and $5 \times 10^{4} \mathrm{cfu}$ in $10 \mu \mathrm{l}$ for $P$. aeruginosa.

The efficacy of four antibiotics: ampicillin, ciprofloxacin, tetracycline and rifampicin, in G. mellonella larvae was assessed against four bacterial pathogens: Gram-positive M. smegmatis and S. aureus, and Gram-negative $E$. coli and $P$. aeruginosa. For each bacterial strain there was at least one antibiotic that is indicated in the treatment of an infection caused by that strain, and at least one that is predicted not to clear the infection to confirm that the antibiotic action, not the insect immunity, is responsible for the recovery.

Antibiotic efficacy testing was carried out as described in "Methods" section. The therapeutic dose of an antibiotic was determined when the antibiotic rescued the mortality caused by the bacterial infection. When even the highest dose of the antibiotic did not clear the infection the antibiotic was regarded as ineffective against those bacteria. The results are summarised in Table 2 . There are four possible outcomes of the antibiotic testing: "(1) predicted dose of an antibiotic clears a bacterial infection, (2) a dose different from the predicted dose clears an infection, (3) antibiotic predicted to be ineffective is ineffective, (4) antibiotic predicted to be ineffective clears the bacterial infection. In most cases the antibiotics performed approximately as predicted, either treating an infection within the dose predicted or being ineffective against a resistant strain.

It was found that ciprofloxacin, tetracycline and rifampicin performed as expected against $E$. coli infections. Both ciprofloxacin and tetracycline worked within the ranges prescribed for humans (10-15 and $25-50 \mathrm{mg} /$ $\mathrm{kg}$ body weight/day respectively). Rifampicin was, as predicted, not effective as Enterobacteriaceae are intrinsically resistant to rifampicin. Ampicillin was expected to clear the infection at $50-200 \mathrm{mg} / \mathrm{kg}$ body weight, but it failed to treat the infection in Galleria. P. aeruginosa was resistant to all antibiotics used. $P$. aeruginosa is intrinsically resistant to ampicillin, tetracycline and rifampicin, and these antibiotics were tested as a negative control. The strain was expected to be sensitive to ciprofloxacin, 


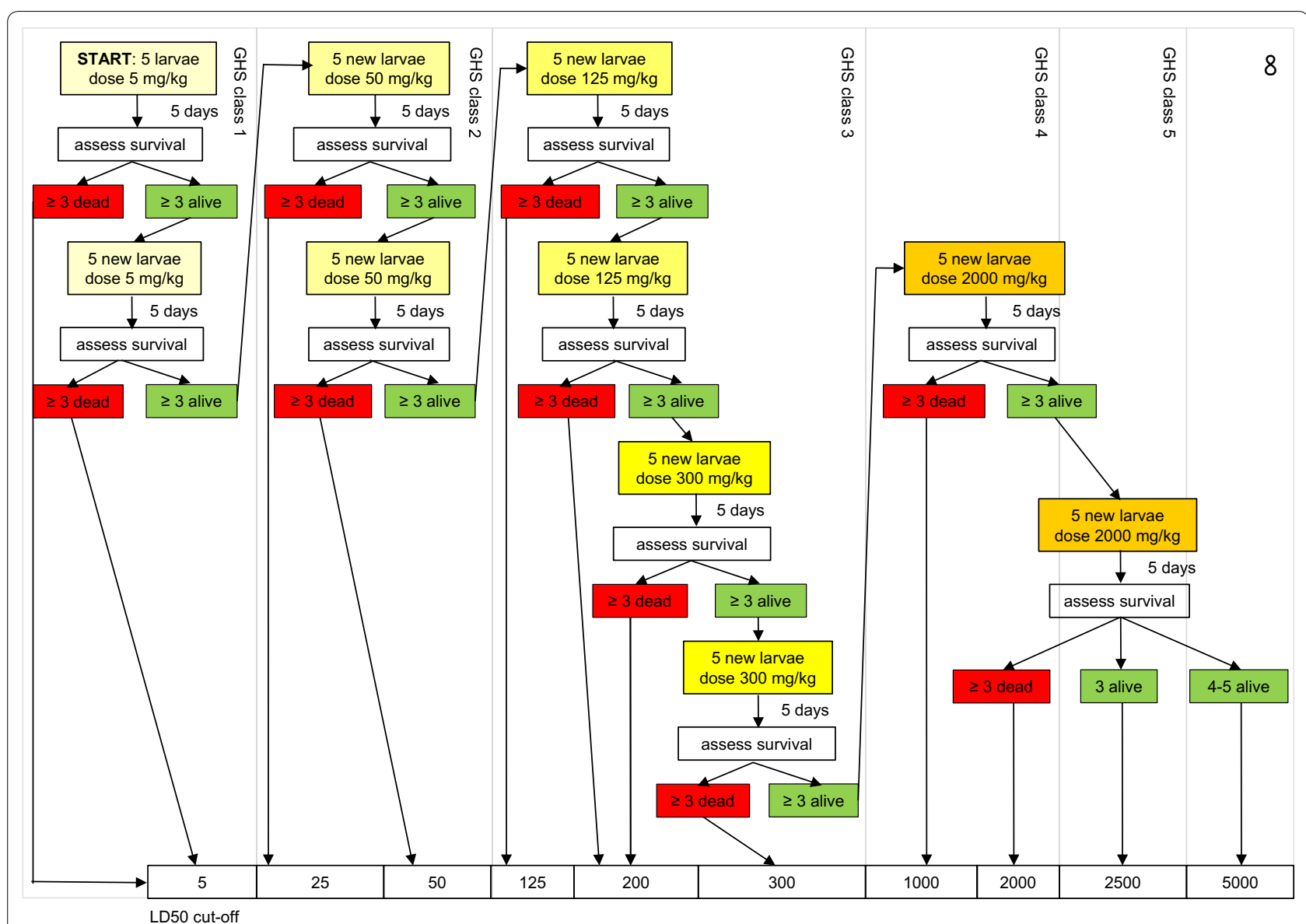

Fig. 2 A flowchart representing consecutive steps in the acute toxicity test. A starting dose of $5 \mathrm{mg} / \mathrm{kg}$ body weight was administered and the insects were scored for mortality. If the mortality was over $40 \%$, the compound was assigned the highest toxicity class. If the mortality is below $40 \%$, the dose was re-tested and the testing continued until a toxic dose was established

Table 2 Predicted and determined antibiotic susceptibility

\begin{tabular}{|c|c|c|c|c|c|c|c|c|}
\hline \multirow[t]{3}{*}{ Bacteria } & \multicolumn{8}{|c|}{ Therapeutic dose (mg/kg body weight) } \\
\hline & \multicolumn{2}{|l|}{ Ampicillin } & \multicolumn{2}{|c|}{ Ciprofloxacin } & \multicolumn{2}{|c|}{ Tetracycline } & \multicolumn{2}{|l|}{ Rifampicin } \\
\hline & Predicted & Test result & Predicted & Test result & Predicted & Test result & Predicted & Test result \\
\hline Escherichia coli & $50-200$ & Resistant & $10-15$ & 25 & $25-50$ & 25 & Resistant & Resistant \\
\hline Mycobacterium smegmatis & Resistant & Resistant & Resistant & Resistant & Resistant & Resistant & $10-20$ & Resistant \\
\hline Pseudomonas aeruginosa & Resistant & Resistant & $10-15$ & Resistant & Resistant & Resistant & Resistant & Resistant \\
\hline Staphylococcus aureus & $50-200$ & 200 & $10-15$ & 200 & Resistant & 50 & $10-20$ & 25 \\
\hline
\end{tabular}

which is active against Gram-negative pathogens, but when tested in the wax moth larvae $P$. aeruginosa was resistant to this drug. M. smegmatis was also resistant to all antibiotics used. Mycobacteria are intrinsically resistant to a range of antibiotics, and ampicillin, ciprofloxacin, and tetracycline were predicted to produce a resistant phenotype. Surprisingly rifampicin did not work, even though it is a standard treatment against mycobacterial infections. All antibiotics used were effective against $S$. aureus. Ampicillin worked at the high end of the spectrum normally prescribed for human use: $50-200 \mathrm{mg} / \mathrm{kg}$ body weight/day. Ciprofloxacin only cleared the infection at a concentration nearly 20 times higher than the dose recommended for human use. Tetracycline was predicted to be ineffective but it cleared the $S$. aureus infection at $50 \mathrm{mg} / \mathrm{kg} /$ body weight. There are a number of potential 
explanations for this, e.g. tetracycline susceptibility could have been caused by the loss of a tetracycline-resistance determinant from the $S$. aureus strain tested. Rifampicin, which is a standard treatment for methicillin-resistant $S$. aureus (MRSA), was effective at a low concentration.

These antibiotic efficacy studies were based on existing literature [2]. G. mellonella larvae have been previously used to study bacterial virulence and susceptibility to antibiotics and our study confirmed that the larvae are a suitable host for antibiotic efficacy studies. Additionally, we determined that the antibiotic therapeutic dose established in G. mellonella often matches the doses recommended for use in humans.

Our experiments support the proposal that antibiotic efficacy testing can be done in G. mellonella and the therapeutic doses recommended for human use can be translated to doses in the wax moth larvae. In most cases the exact dose recommended for clinical treatment of a systemic infection can be calculated for larval body weight and can clear an infection. Such correlation is possible because often the mechanisms of microbial virulence are not host-specific. Where the predictions did not match the outcomes, there are a variety of potential explanations, e.g. differences in immune responses in different organisms, etc. Previous studies have shown that the fungal pathogen Candida albicans uses the same repertoire of effectors, involved in fungal virulence and yeastto-hypha transition, against insects and mammals [4]. Similarly, the bacterial pathogen $P$. aeruginosa employs a similar set of virulence genes to overcome the immune system of wax moth larvae and mice [6] and the larvae can be used to identified virulence factors required for an infection in mammals.

\section{Toxicity testing}

11 compounds were provided by Inspiralis Ltd. to test for their toxicity in G. mellonella larvae. The compounds were initially tested blind, i.e. they were supplied in numbered tubes and only after the test procedure was completed were the numbers linked to compound names. The aim of this approach was to avoid bias, i.e. assigning lower toxic doses to known toxic compounds and higher ones to safer compounds.

Insects (5-10 larvae) were injected with $10 \mu \mathrm{l}$ compound into the hindmost proleg. Intraperitoneal injection was used in the procedures to strictly control the amount of toxic compound or bacterial pathogen that the larvae were exposed to. Alternative approaches, not applied in this study, use feeding procedures [16] or contact exposure [17]. The quantification of exposure to a compound is less precise for such procedures, but it is sometimes a more appropriate method when a route of exposure is known. For example, pesticide toxicity in insects testing normally employs feeding studies [18] as it is the normal route of exposure.

Insects were injected with low doses $(5 \mathrm{mg} / \mathrm{kg}$ body weight) of the compounds first and the mortality was recorded daily for 5 days. If no mortality was observed new groups of larvae were injected with compounds at $50 \mathrm{mg} /$ $\mathrm{kg}$ body weight and the mortality was recorded daily for 5 days again. When mortality was observed in $60 \%$ or more of the larvae, the compound was re-tested at that same concentration to confirm the toxicity. When mortality below $60 \%$ was observed, the compounds were tested subsequently at 125,300 and $2000 \mathrm{mg} / \mathrm{kg}$ body weight. Each compound was assigned an $\mathrm{LD}_{50}$ value (Table 3 ) and the values were compared to toxicity data available in the MSDS pages. No compounds were tested above $2000 \mathrm{mg} /$ $\mathrm{kg}$ body weight in line with OECD guidelines [15]. Such high compound concentrations introduce solubility issues and are discouraged in the guidelines as unnecessary and unethical. Generally, compounds with no indication of toxicity at $2000 \mathrm{mg} / \mathrm{kg}$ body weight are considered non-toxic.

\section{Comparison of toxicity testing in G. mellonella with studies in rodents}

There are three possible outcomes of the comparison of toxicity in the wax moth larvae and in rodents. Firstly, the toxicity can be the same or very similar. Secondly the toxic doses in G. mellonella can be higher than the ones in rodents, and finally the toxic doses in G. mellonella can be lower than the doses for rodents. Our standard injection medium, 50\% DMSO in water, was lethal to wax moths at a dose equivalent to $100 \mathrm{mg} / \mathrm{kg}$ body weight (a $4 \mu \mathrm{l}$ injection of a stock solution or more than $2 \mu \mathrm{l}$ of pure DMSO per injection). All compounds toxic above $100 \mathrm{mg} / \mathrm{kg}$ body weight had to be re-tested in a modified injection medium with a decreased amount of DMSO $(<10 \%$ final; Table 3$)$.

For most compounds the toxicity determined in this experiment correlates well with toxicity reported in the MSDS pages available for each of the compounds. The compounds with the lowest $\mathrm{LD}_{50} \mathrm{~s}$ in mammals were the most toxic for the wax moth larvae (Table 3). The most toxic compounds had the lowest $\mathrm{LD}_{50}$ values: the value for doxorubicin fell within the range established by experiments in mammals $(5.5 \mathrm{mg} / \mathrm{kg}$ in Galleria vs 1.2 and $16 \mathrm{mg} / \mathrm{kg}$ in mice and rats respectively) and for amsacrine the value was lower $(40 \mathrm{mg} / \mathrm{kg}$ via intraperitoneal injection vs $100 \mathrm{mg} / \mathrm{kg}$ in rats and $243 \mathrm{mg} / \mathrm{kg}$ in mice via oral exposure). Similar correlations between $\mathrm{LD}_{50}$ values established in G. mellonella and $\mathrm{LD}_{50}$ values from MSDS pages was also observed for etoposide, ciprofloxacin, and streptomycin.

Glucose, sodium chloride and tetracycline were not toxic to G. mellonella at $2000 \mathrm{mg} / \mathrm{kg}$ body weight, and in 
Table 3 Toxicity of compounds used in the trial

\begin{tabular}{|c|c|c|c|c|c|}
\hline \multirow[t]{3}{*}{ Compound } & \multicolumn{5}{|c|}{$\mathrm{LD}_{50}(\mathrm{mg} / \mathrm{kg} \text { body weight })^{\mathrm{a}}$} \\
\hline & \multirow{2}{*}{$\begin{array}{l}\text { G. mellonella } \\
\text { Intraperitoneal }\end{array}$} & \multicolumn{2}{|l|}{ Rat } & \multicolumn{2}{|l|}{ Mouse } \\
\hline & & Oral & Intraperitoneal & Oral & Intraperitoneal \\
\hline Amsacrine & 40 & 100 & & 243 & \\
\hline Chloroquine & $125^{b}$ & 623 & & 500 & \\
\hline Ciprofloxacin & $>2000$ & $>2000$ & & $>2000$ & \\
\hline DMSO & 100 & 14,500 & & 7920 & \\
\hline Doxorubicin & 5.5 & & 16 & 698 & 1.2 \\
\hline Etoposide & 100 & 1784 & 58 & 215 & \\
\hline Glucose & $>2000$ & 25,800 & & & \\
\hline Novobiocin & 100 & 3500 & & 962 & \\
\hline Sodium chloride & $>2000$ & 3000 & & 4000 & \\
\hline Streptomycin & 300 & 430 & & 430 & \\
\hline Tetracycline & $>2000$ & 6443 & 318 & 2759 & 368 \\
\hline
\end{tabular}

${ }^{a} \mathrm{LD}_{50}$ values determined in the test were compared to values available in Material Safety Data sheets provided with the compounds. Blank space indicates the data are not available

b Compounds tested at $>100 \mathrm{mg} / \mathrm{Kg}$ were initially tested in 50\% DMSO (which is toxic to G. mellonella) and then re-tested at lower [DMSO]s such that the final [DMSO] was $10 \%$

line with the guidelines for toxicity testing in murine models, the compounds were not tested above this concentration. The $\mathrm{LD}_{50}$ values established in rats and mice were higher than $2000 \mathrm{mg} / \mathrm{kg}$ body weight. The values obtained for novobiocin and chloroquine were thirty to ten times smaller than the mammalian $\mathrm{LD}_{50}$, but the only data available is from oral exposure, making the values difficult to compare. The same is true for $50 \% \mathrm{DMSO}$, which is more toxic to G. mellonella larvae than to rats and mice.

An important limitation of using G. mellonella larvae in toxicity testing, which also applies to all other toxicity testing systems, is that the toxicity cannot be directly tested in humans and it is not known how experimental $L D_{50}$ values correlate to human values or even if the mechanisms of toxicity are the same. There are numerous mechanisms of systemic toxicity and they are poorly understood. In some cases, the cause of toxicity is alike in different systems. For example, doxorubicin is a DNA intercalator and poisons different organisms at low doses.

The use of DMSO as an injection medium in the procedure is another limitation to the study. 50\% DMSO was lethal to wax moths above $100 \mathrm{mg} / \mathrm{kg}$ body weight when used as an injection medium. The test cannot correctly assign $\mathrm{LD}_{50}$ to mildly toxic compounds in 50\% DMSO (for example antibiotics ciprofloxacin and chloramphenicol) and non-toxic compounds (glutamic acid, glucose, sodium chloride) because of the side effects of the DMSO injection. Decreasing the amount of DMSO per injection (to $10 \%$ or less), maintains the solubility of the compounds tested without compromising the test procedure. Alternatively, the solvent effects can be subtracted from the compound effects using statistical methods. Restricting background mortality (mortality in untreated control groups) not only lowers the experimental noise but also aligns better with the guidelines on the use of laboratory animals.

Overall our experiments have shown that Galleria larvae can be used in acute toxicity testing, providing data more cheaply and quickly than traditional testing systems. Testing in G. mellonella is unlikely to fully replace toxicity testing in mammals, but it is a convenient step between in vitro tests and testing in mammals, adding more complexity to the former and statistical robustness to the latter.

\section{Conclusion}

In summary, our experiments support that proposal that antibiotic efficacy can be tested in vivo in Galleria mellonella larvae. We established that the doses recommended for use in humans can be effective in systemic infections in the larvae and that the acute toxicity of compounds in wax moth larvae correlates to the toxicity in mice and rats. G. mellonella is an organism that can be easily adopted in various tests. It cannot fully replace mammalian models, but it is much cheaper and can provide the statistical robustness current animal models lack.

\section{Abbreviations}

DMSO: dimethyl sulfoxide; MSDS: material safety data sheet; PBS: phosphate-buffered saline; OECD: Organisation for Economic Co-operation and Development. 


\section{Authors' contributions}

$\mathrm{KI}$ and $\mathrm{AM}$ conceived and designed the experiments; $\mathrm{KI}$ performed the experiments and analysed the data; KI and AM wrote the paper. Both authors read and approved the final manuscript.

\section{Acknowledgements}

We thank lan Bedford, Anna Jordan and Jake Stone (John Innes Centre Entomology Facility) for their assistance with insect rearing. We thank Nick Burton Lindsay Hall and Martin Stocks for their comments on the manuscript.

\section{Competing interests}

The authors declare that they have no competing interests.

\section{Availability of data and materials}

All data is given in the main body of the manuscript; materials are available from the authors.

\section{Compliance with ethical guidelines}

Not applicable.

\section{Consent to publish}

Not applicable.

\section{Consent to participate}

Not applicable.

\section{Funding}

KI was supported by a BBSRC-CASE studentship from BBSRC, Inspiralis Ltd. and Plant Bioscience Ltd. Work in A.M.'s lab is also supported by grant BB/ J004561/1 from BBSRC (UK) and the John Innes Foundation.

\section{Publisher's Note}

Springer Nature remains neutral with regard to jurisdictional claims in published maps and institutional affiliations.

Received: 4 January 2017 Accepted: 23 August 2017

Published online: 29 August 2017

\section{References}

1. Graham ML, Prescott MJ. The multifactorial role of the $3 R$ in shifting the harm-benefit analysis in animal models of disease. Eur J Pharmacol. 2015;759:19-29.

2. Cook SM, McArthur JD. Developing Galleria mellonella as a model host for human pathogens. Virulence. 2013;4(5):350-3.
3. Thomas RJ, Hamblin KA, Armstrong SJ, Muller CM, Bokori-Brown M, Goldman S, Atkins HS, Titball RW. Galleria mellonella as a model system to test the pharmacokinetics and efficacy of antibiotics against Burkholderia pseudomallei. Int J Antimicrob Agents. 2013;41(4):330-6.

4. Brennan M, Thomas DY, Whiteway M, Kavanagh K. Correlation between virulence of Candida albicans mutants in mice and Galleria mellonella larvae. FEMS Immunol Med Microbiol. 2002;34(2):153-7.

5. Fuchs BB, O'Brien E, Khoury JB, Mylonakis E. Methods for using Galleria mellonella as a model host to study fungal pathogenesis. Virulence. 2010;1(6):475-82.

6. Jander G, Rahme LG, Ausubel FM. Positive correlation between virulence of Pseudomonas aeruginosa mutants in mice and insects. J Bacteriol. 2000;182(13):3843-5.

7. Cytrynska M, Zdybicka-Barabas A, Jakubowicz T. The involvement of protein kinase A in the immune response of Galleria mellonella larvae to bacteria. Acta Biochim Pol. 2007:54(1):167-74.

8. Browne N, Heelan M, Kavanagh K. An analysis of the structural and functional similarities of insect hemocytes and mammalian phagocytes. Virulence. 2013;4(7):597-603.

9. Peleg AY, Jara S, Monga D, Eliopoulos GM, Moellering RC Jr, Mylonakis E. Galleria mellonella as a model system to study Acinetobacter baumannii pathogenesis and therapeutics. Antimicrob Agents Chemother. 2009;53(6):2605-9.

10. Harding CR, Schroeder GN, Collins JW, Frankel G. Use of Galleria mellonella as a model organism to study Legionella pneumophila infection. J Vis Exp JoVE. 2013;81:e50964.

11. Megaw J, Thompson TP, Lafferty RA, Gilmore BF. Galleria mellonella as a novel in vivo model for assessment of the toxicity of 1-alkyl-3-methylimidazolium chloride ionic liquids. Chemosphere. 2015:139:197-201.

12. Desbois AP, Coote PJ. Wax moth larva (Galleria mellonella): an in vivo model for assessing the efficacy of antistaphylococcal agents. J Antimicrob Chemother. 2011;66:1785-90.

13. Hill L, Veli N, Coote PJ. Evaluation of Galleria mellonella larvae for measuring the efficacy and pharmacokinetics of antibiotic therapies against Pseudomonas aeruginosa infection. Int J Antimicrob Agents. 2014;43(3):254-61.

14. Szczeklik A, Gajewski P. Interna Szczeklik 2014. Textbook of internal medicine in 2014. 2014.

15. OECD. Guidance document on acute oral toxicity testing. OECD Publishing; 2001.

16. OECD. Test No. 237: Honey bee (Apis mellifera) larval toxicity test, single exposure. OECD Publishing; 2013

17. OECD. Test No. 214: Honeybees, acute contact toxicity test. OECD Publishing; 1998.

18. Charbonneau C, Cote R, Charpentier G. Effects of azadirachtin and of simpler epoxy-alcohols on survival and behaviour of Galleria mellonella (Lepidoptera). J Appl Entomol. 2007;131(7):447-52.

\section{Submit your next manuscript to BioMed Central and we will help you at every step:}

- We accept pre-submission inquiries

- Our selector tool helps you to find the most relevant journal

- We provide round the clock customer support

- Convenient online submission

- Thorough peer review

- Inclusion in PubMed and all major indexing services

- Maximum visibility for your research

Submit your manuscript at www.biomedcentral com/submit
Biomed Central 